\title{
Investigation on Elderly Health Situation in Yan'an City
}

\author{
Shangwu Tao ${ }^{1}$ \\ ${ }^{1}$ College of Physical Education, Yan'an University, Yan'an, China
}

Keywords: Yan'an city; elderly; fitness; Investigation.

\begin{abstract}
Through the literature, questionnaire survey, mathematical statistics. Yan'an City, the elderly in the elderly to participate in the status quo of the investigation and found that women in the fitness of the elderly was significantly more than men, the elderly to participate in fitness projects mainly to soothe the main strength and other characteristics. It is suggested that schools and communities should conduct reasonable networking, increase the training of social sports personnel, promote cooperation between schools and communities, and strengthen the development and construction of sports venues.
\end{abstract}

\section{Introduction}

Elderly people are an important part of social groups, the health of the elderly has been a concern. With the development of the aging population, the elderly this group was gradually expanding the trend, how to make the elderly healthy, happy and happy to enjoy the old age has been the problem of people thinking. The elderly with age, body organs gradually aging, if they can participate in the movement of the law, will enable the elderly to fully play the limb function, improve the health of the elderly, reduce the disease, improve the quality of life, in order to facilitate the elderly Mental health[1]. This paper investigates the present situation of the elderly in Yan'an City by observing and studying the current situation and existing problems of the elderly in Yan'an city, and puts forward the corresponding measures to solve the problem and make the elderly more healthy and happy life.

\section{Research object and method}

Object of study. This paper takes the present situation of the elderly in Yan'an city as the research object, and randomly sampled 300 elderly people who participated in physical exercise in Yan'an city by random sampling method.

Research methods. Literature method. Search by CNKI, Wanfang database, browse and read the relevant papers.

Questionnaire survey. There were 300 questionnaires, 280 replies, 93\% recovery, 265 valid questionnaires, 95\% effective rate, and 169 women in the squares, communities and sports venues where the elderly in the urban area of the city of Yan'an City, Male 96.

\section{Results and analysis}

Participate in the sex and age survey of the elderly.

Table 1 Participate in fitness for older sex and age structure $(\mathrm{N}=256)$

\begin{tabular}{cccccc}
\hline category & male & female & $60-70$ years & $70-80$ years & Above 80 years old \\
\hline The number of & 96 & 169 & 170 & 88 & 7 \\
percentage(\%) & 36 & 64 & 64.1 & 33.2 & 2.7 \\
\hline
\end{tabular}


Table 1 shows that the number of women participating in physical exercise is far greater than that of men. Analysis of the reasons: the popularity of the square dance so that many women find their favorite sports, so that they like the physical exercise; Women 's physical exercise can promote good health, enhance immunity, anti - aging awareness higher than men; Women like the collective social, many elderly people gathered together while chatting to meet the lonely inner needs of the elderly. 60-70 years old to participate in physical exercise of the largest proportion of the elderly, 70-80 years old in the middle of the elderly over the age of 80 the smallest proportion, the reason for this situation may be 60-70 years old to recognize Physical exercise to enhance immunity, the importance of anti-aging, so actively engaged in physical exercise, but with age, organ aging, to 80 years old, frail, mobility, not suitable for physical exercise.

Elderly participation in fitness when the sports selection survey.

$\underline{\text { Table } 2 \text { List of selection of sports items for the elderly during physical exercise }(\mathrm{n}=265)}$

\begin{tabular}{ccc} 
Sports items & The number of & Percentage $(\%)$ \\
\hline Walk, run & 59 & 22.3 \\
Taiji boxing & 63 & 23.8 \\
Shuttlecock & 16 & 6 \\
Square dance & 73 & 27.6 \\
Yangge & 29 & 10.9 \\
Other & 25 & 9.4 \\
\hline
\end{tabular}

According to Table 2 survey results show: Yan'an city elderly people to participate in the selection of physical exercise projects on the more diversified. Older people choose square dance, walking the most number of walking, because these actions from time, site constraints, easy to carry out, and exercise good results. Choose tai chi the elderly are more, because the tai chi action soothing, and able to self-cultivation, cultivate sentiment. Yangge as a unique folk traditional culture in northern Shaanxi, Yan'an City, the elderly is the choice of exercise. Choosing shuttlecock of the elderly is better physical, older age is also relatively small.

Elderly participates in fitness frequency and time of investigation. Physical exercise is particularly important for the elderly, it can not only enhance the elderly immune system and the elderly cardiovascular and cerebrovascular function, but also make the elderly slow down the aging. Through Table 3 shows that most of the elderly in Yan'an City, the elderly or pay more attention to physical exercise, exercise time and frequency can basically meet the health standards. But there are some elderly people lack adequate attention to physical exercise, that participate in physical exercise has no effect, a waste of time to participate in physical exercise, the elderly accounted for $8.7 \%$. In the survey of the elderly, $47.9 \%$ of the elderly each time to participate in physical exercise time in $30 \mathrm{~min}$, $52.1 \%$ of the elderly each time to participate in physical exercise time in 30-60min, the proportion of the two roughly the same, And Yan'an City Pagoda District urban elderly exercise time choices are in the morning and evening. Although the elderly in Yan'an City, the intensity of physical exercise, frequency and so generally meet the requirements of the elderly physical exercise, but the vast majority of the elderly in the physical exercise are free, there is no general plan, which is Yan'an City pagoda The lack of physical exercise in the elderly in the urban area. 
Table 3 Elderly fitness frequency and time

\begin{tabular}{cccccc}
\hline & \multicolumn{3}{c}{ Exercise times } & \multicolumn{2}{c}{ Exercise time } \\
\cline { 2 - 6 } & $\begin{array}{c}\text { Little } \\
\text { involvement }\end{array}$ & $\begin{array}{c}3 \text { times a } \\
\text { week }\end{array}$ & $\begin{array}{c}\text { More than } 3 \\
\text { times }\end{array}$ & $\begin{array}{c}\text { Below } \\
30 \mathrm{~min}\end{array}$ & $\begin{array}{c}\text { More than } \\
30 \mathrm{~min}\end{array}$ \\
\hline $\begin{array}{c}\text { The number of } \\
\text { Percentage } \\
(\%)\end{array}$ & 23 & 96 & 146 & 127 & 138 \\
\hline
\end{tabular}

Scientific research shows that scientific physical exercise can promote good health, the elderly cardiovascular and cerebrovascular, physical function enhancement has a great effect[2]. Most of the elderly are able to recognize this point, but there are some elderly people lack the scientific awareness of exercise, will not reasonably arrange exercise intensity, intensity is too small, less than the expected exercise effect, the intensity is too large, played the opposite Exercise effect, the elderly to participate in physical exercise when the body sweating is the best effect. So the urban area of Yan'an City, most of the elderly physical strength is to meet the requirements.

Survey of fitness places and instructors in the elderly. Most of the elderly have chosen square parks and community vacations for physical exercise, probably because the square park and the community space are the gathering place for the elderly, and many elderly people participate in physical exercise in a good atmosphere[3]. In the investigation process found: Yan'an City, the elderly physical exercise places limited, there are security risks. Mainly in the provision of physical exercise for the elderly less places, some elderly people choose to go on the road for jogging or walking; the square community of exercise equipment lack of maintenance, there are security risks; the square is not optimistic about the environment, some traders In the pendulum, can not provide the elderly a clean, relaxed exercise environment, not a good exercise effect. In place of each exercise, there is no professional social sports instructors to guide the elderly physical exercise, the elderly most of their choice according to their own sports, there is no scientific system targeted for physical exercise, so that no better exercise effect.

Health, entertainment, fitness and disease prevention is the main purpose of Yanan city elderly to participate in physical exercise, in which there are some old people's choice of entertainment, through the analysis of these old people have retired at home, some children work outside their home in the side, lonely, lack of companionship, so choose to square and other old people chat, square dance sports to fill the inner loneliness, pass the time.

\section{Conclusion and suggestion}

Conclusion. Yan'an city elderly people in the fitness frequency and the distribution of the time used to show a considerable phenomenon, but there are still some elderly people in the movement frequency interval is longer, less than 3 times per week and each fitness time less than 30 minutes , The two phenomena coexist. Yan'an City, the elderly to participate in the exercise of the elderly in the form of diversification, will promote the health and disease prevention as the primary goal of the majority of the crowd. Elderly physical exercise selected by the type of project is mainly concentrated in those who are not subject to space, equipment and other hardware facilities limit the project, but also a single project, continue to choose this type of project will reduce the crowd is not conducive to the elderly The construction of an atmosphere of physical exercise. Yan'an City, the elderly in the city to participate in physical training to choose the venue, the neighborhood near the park square and the community simple fitness places, because these public fitness places are relatively fixed, convenient and do not charge any fees, and choose to exercise in the commercial gym Older people at least. 
Suggestion. Schools and communities to carry out a reasonable friendship, play Yan'an City college graduates on social sports guidance personnel investment. Relevant departments should strengthen cooperation with sports associations, so that long-term participation in physical exercise of the crowd to join the formation of their own characteristics of the group, to strengthen the group and the exchange of external groups[4]. Promote the cooperation of schools and communities, through lectures, exchanges and other forms of the elderly in the fitness project selection on the scientific guidance, while strengthening the sports venues development and construction.

\section{References}

[1] Wang Zhongshan,A Study on the Effects of Blood Rheology and Hemorheology in Elderly Patients of Taijiquan, Journal of Tianjin Physical Education Institute,vol.1,pp.51-53, 1999

[2] Bi Huawei and so on, Analysis of the main factors affecting the development of community elderly Sports, Shandong Sports Science and Technology,vol.3,pp79-81,2005

[3] Pei Lixin,An Analysis of the Current Situation of Aging in China and the Research on the Countermeasure of the Aged, Sports science, vol.3,pp23-27,2001

[4] Lu Yongmei et al,Research progress of fitness in the elderly , Sports and science,vol.20,pp. 9-15,1999. 